\title{
Influence of Slip Condition on Unsteady Free Convection Flow of Viscous Fluid with Ramped Wall Temperature
}

\author{
Sami Ul Haq, ${ }^{1}$ Ilyas Khan, ${ }^{2}$ Farhad Ali, ${ }^{1}$ Arshad Khan, ${ }^{3}$ and Tarek Nabil Ahmed \\ Abdelhameed ${ }^{2,4}$ \\ ${ }^{1}$ Department of Mathematics, City University of Science and Information Technology, Peshawar 25000, Pakistan \\ ${ }^{2}$ Department of Basic Sciences, College of Engineering Majmaah University, P.O. Box 66, Majmaah 11952, Saudi Arabia \\ ${ }^{3}$ Department of Mathematical Sciences, Faculty of Science, Universiti Teknologi Malaysia (UTM), 81310 Skudai, Malaysia \\ ${ }^{4}$ Mathematics Department, Faculty of Science, Beni-Suef University, Beni-Suef 62514, Egypt
}

Correspondence should be addressed to Sami Ul Haq; samiulhaqmaths@yahoo.com

Received 27 June 2014; Revised 16 November 2014; Accepted 24 November 2014

Academic Editor: Saeed Islam

Copyright (c) 2015 Sami Ul Haq et al. This is an open access article distributed under the Creative Commons Attribution License, which permits unrestricted use, distribution, and reproduction in any medium, provided the original work is properly cited.

The objective of this study is to explore the influence of wall slip condition on a free convection flow of an incompressible viscous fluid with heat transfer and ramped wall temperature. Exact solution of the problem is obtained by using Laplace transform technique. Graphical results to see the effects of Prandtl number Pr, time $t$, and slip parameter $\eta$ on velocity and skin friction for the case of ramped and constant temperature of the plate are provided and discussed.

\section{Introduction}

Free convection flow occurs due to a buoyancy-induced motion resulting from the body forces acting on density gradients and is particularly important in atmospheric and oceanic circulation, in the problems of heat rejection and removal in many devices, in the design of spaceships and filtration process. Siegel [1], in his pioneering work studied the unsteady free convection flow past a semi-infinite vertical plate with uniform temperature. However, many practical problems usually require wall conditions which are nonuniform or arbitrary. In order to understand such problems it is important to investigate problems subject to a step change in the wall temperature. An early attempt was made by Schetz [2] by developing an approximate analytical model. Later Hayday et al. [3] used a numerical approach. Of these works, Malhotra et al. [4] mentioned that in the fabrication of thinfilm photovoltaic devices ramped wall temperatures can be employed to control the temperature uniformity of the system. Periodic temperature step changes are also important in building heat transfer applications, for example, in air conditioning, where the conventional assumption of periodic outdoor conditions may lead to considerable errors in the case of a significant temporary deviation of the temperature from periodicity, as discussed by Antonopoulos and Democritou [5]. Due to the aforementioned significance of step change in the wall temperature Chandran et al. [6] presented an analytical solution to the unsteady natural convection flow of an incompressible viscous flow near a vertical plate with ramped wall temperature. Seth et al. [7] elaborated the unsteady hydromagnetic natural convection flow of a viscous incompressible electrically conducting fluid with radiative heat transfer near an impulsively moving vertical flat plate embedded in a porous medium with ramped wall temperature. Narahari et al. [8] investigated mass transfer effects on free convection flow past an infinite vertical plate subject to discontinuous or nonuniform wall temperature conditions.

The above achievements are made with the assumption of no-slip condition between the wall and the fluid. The effects of fluid slippage at the wall appear in many applications such as in microchannels or nanochannels and in applications where a thin film of light oil is attached to the moving plates or when the surface is coated with special coatings such as thick monolayer of hydrophobic octadecyltrichlorosilane [9]. The fluid problem in the slip flow regime is very important in the era of modern science, technology, and vast ranging 
industrialization. In view of such applications, Makinde and Osausi [10] studied the combined effect of magnetic field and permeable wall slip velocity on the steady flow of an electrically conducting fluid in a channel of uniform width. Makinde and Mhone [11] investigated the combined effect of a transverse magnetic field and radiative heat transfer to unsteady flow of a conducting optically thin fluid through a channel filled with saturated porous medium and nonuniform walls temperature. Mehmood and Ali [12] extended the work of Makinde and Mhone [11] by considering the fluid slip at the lower wall. Few other attempts taking into account the slip boundary condition are [13-16]. However, the literature lacks studies that take into consideration the combined effect of slippage and ramped temperature at the wall on the unsteady free convection flow of a viscous incompressible fluid near a vertical flat plate. This is the source of motivation to study the influence of slip condition on the unsteady free convection transient flow near a vertical flat plate with ramped wall temperature.

\section{Formulation of the Problem and Solution}

Let us consider the flow of an incompressible viscous fluid near an infinite vertical plate. The $x^{\prime}$-axis is taken along the wall in the upward direction and $y^{\prime}$-axis is taken perpendicular to it into the fluid. At the initial moment $t^{\prime}=0$, both the plate and the fluid are at rest at a constant temperature $T_{\infty}^{\prime}$. At time $t^{\prime}=0^{+}$, the temperature of the plate is raised or lowered to $T_{\infty}^{\prime}+\left(T_{w}^{\prime}-T_{\infty}^{\prime}\right)\left(t^{\prime} / t_{0}\right)$, and then, for $t^{\prime}>t_{0}$, the temperature is maintained at the constant temperature $T_{w}^{\prime}$. In view of the above assumptions, as well as of the usual Boussinesq's approximation, the governing equations reduce to those obtained by Chandran et al. [6, Equations (1) and (2)]:

$$
\begin{gathered}
\frac{\partial u^{\prime}}{\partial t^{\prime}}=\nu \frac{\partial^{2} u^{\prime}}{\partial y^{\prime 2}}+g \beta\left(T^{\prime}-T_{\infty}^{\prime}\right), \\
\frac{\partial T^{\prime}}{\partial t^{\prime}}=\frac{k}{\rho c_{p}} \frac{\partial^{2} T^{\prime}}{\partial y^{\prime 2}}
\end{gathered}
$$

where $u^{\prime}, T^{\prime}, \nu, \rho, g, \beta, k$, and $c_{p}$ are, respectively, the velocity in the $x^{\prime}$ direction, temperature of the fluid, kinematic viscosity, fluid density, acceleration due to gravity, volumetric coefficient of thermal expansion, thermal conductivity, and specific heat at constant pressure.

The appropriate initial and boundary conditions are

$$
\begin{aligned}
& u^{\prime}\left(y^{\prime}, 0\right)=0, \quad T^{\prime}\left(y^{\prime}, 0\right)=T_{\infty}^{\prime} \quad \text { for } y^{\prime} \geq 0, \\
& u^{\prime}\left(0, t^{\prime}\right)-\lambda \frac{\partial u^{\prime}\left(0, t^{\prime}\right)}{\partial y^{\prime}}=0, \quad \text { for } t^{\prime}>0, \\
& T^{\prime}\left(0, t^{\prime}\right)= \begin{cases}T_{\infty}^{\prime}+\left(T_{w}^{\prime}-T_{\infty}^{\prime}\right) \frac{t^{\prime}}{t_{0}} & \text { for } 0<t^{\prime} \leq t_{0} \\
T_{w}^{\prime} & \text { for } t^{\prime}>t_{0},\end{cases}
\end{aligned}
$$

$$
\begin{array}{r}
u^{\prime}\left(y^{\prime}, t^{\prime}\right) \longrightarrow 0, \quad T^{\prime}\left(y^{\prime}, t^{\prime}\right) \longrightarrow T_{\infty}^{\prime} \\
\text { as } y^{\prime} \longrightarrow \infty \text { for } t^{\prime}>0 .
\end{array}
$$

Introducing the following dimensionless variables

$$
\begin{array}{ll}
u=\sqrt{\frac{t_{0}}{v}} u^{\prime}, \quad y=\frac{1}{\sqrt{v t_{0}}} y^{\prime}, \quad t=\frac{t^{\prime}}{t_{0}}, \\
\theta=\frac{T^{\prime}-T_{\infty}^{\prime}}{T_{w}^{\prime}-T_{\infty}^{\prime}}, \quad \operatorname{Pr}=\frac{\rho v c_{p}}{k}, \quad \eta=\frac{\lambda}{\sqrt{v t_{0}}}
\end{array}
$$

and dropping out the prime notation from $u, y$, and $t$, the governing equations (1) take the simplified forms

$$
\begin{gathered}
\frac{\partial u(y, t)}{\partial t}=\frac{\partial^{2} u(y, t)}{\partial y^{2}}+\theta(y, t) ; \quad y, t>0, \\
\operatorname{Pr} \frac{\partial \theta(y, t)}{\partial t}=\frac{\partial^{2} \theta(y, t)}{\partial y^{2}} ; \quad y, t>0,
\end{gathered}
$$

where $\operatorname{Pr}=\rho v c_{p} / k$ is the Prandtl number. According to the above nondimensionalisation process, the characteristic time $t_{0}$ can be defined as

$$
t_{0}=\left[\frac{v}{g^{2} \beta^{2}\left(T_{w}^{\prime}-T_{\infty}^{\prime}\right)^{2}}\right]^{1 / 3} .
$$

In dimensionless form, the initial and boundary conditions (2) become

$$
\begin{gathered}
u(y, 0)=0, \quad \theta(y, 0)=0 \quad \text { for } y \geq 0, \\
u(0, t)-\eta \frac{\partial u(0, t)}{\partial y}=0, \quad \text { for } t>0, \\
\theta(0, t)= \begin{cases}t & \text { for } 0<t \leq 1 \\
1 & \text { for } t>1,\end{cases} \\
u(y, t) \longrightarrow 0, \quad \theta(y, t) \longrightarrow 0 \\
\text { as } y \longrightarrow \infty \quad \text { for } t>0,
\end{gathered}
$$

where $\eta$ is the dimensionless slip parameter.

Equations (4) are a coupled linear system of equations, which can be solved by the Laplace transform technique subject to the initial and boundary conditions (6). The solutions of energy and momentum equations are

$$
\theta(y, t)=F(y, t)-F(y, t-1) H(t-1),
$$

$$
\begin{array}{r}
u(y, t) \\
=\left\{\begin{array}{rr}
\frac{1}{\operatorname{Pr}-1}\left[u_{1}(y, t)-u_{2}(y, t)+\sqrt{\operatorname{Pr}} u_{3}(y, t)\right. \\
-\left\{u_{1}(y, t-1)-u_{2}(y, t-1)\right. \\
\left.+\sqrt{\operatorname{Pr}} u_{3}(y, t-1)\right\} & H(t-1)] \\
& \text { for } \operatorname{Pr} \neq 1 \\
u_{3}(y, t)+u_{4}(y, t) & \text { for } \operatorname{Pr}=1 \\
-\left\{u_{3}(y, t-1)+u_{4}(y, t-1)\right\} & H(t-1)
\end{array}\right.
\end{array}
$$


where

$$
\begin{aligned}
& F(y, t)=\left(\frac{\operatorname{Pr} y^{2}}{2}+t\right) \operatorname{erfc}\left(\frac{y \sqrt{\operatorname{Pr}}}{2 \sqrt{t}}\right) \\
& -\sqrt{\frac{\operatorname{Pr} t}{\pi}} y \exp \left(\frac{\operatorname{Pr} y^{2}}{4 t}\right), \\
& u_{1}(y, t)=t^{2} \exp \left(-\frac{a y^{2}}{2}\right) \sinh \left(\frac{a y}{2}\right) \\
& +\frac{t^{2}}{2} \exp \left(-a y^{2}\right)-\frac{a}{\pi} \\
& \times \int_{0}^{t} \int_{0}^{\infty}\left(\frac{1-e^{-x s}}{x^{2}}\right) \\
& \times\left[\frac{a \sin (y \sqrt{x})}{x+a^{2}}+\frac{\sqrt{x} \cos (y \sqrt{x})}{x+a^{2}}\right] d x d s, \\
& u_{2}(y, t)=\frac{1}{2}\left(\frac{\operatorname{Pr}^{2} y^{4}}{12}+\operatorname{Pr} y^{2} t+t^{2}\right) \operatorname{erfc}\left(\frac{y \sqrt{\operatorname{Pr}}}{2 \sqrt{t}}\right) \\
& -\sqrt{\frac{\operatorname{Pr} t}{\pi}} \frac{y}{6} \exp \left(\frac{\operatorname{Pr} y^{2}}{2}+5 t\right) \exp \left(-\frac{\operatorname{Pr} y^{2}}{4 t}\right), \\
& u_{3}(y, t)=\frac{1}{3 a \sqrt{\pi}} \\
& \times\left[\sqrt{\pi} y^{3}+\sqrt{t}\left(4 t-2 y^{2}\right) e^{-y^{2} / 4 t}\right. \\
& \left.-\sqrt{\pi} y^{3} \operatorname{erfc}\left(\frac{y}{2 \sqrt{t}}\right)\right]-\left(\frac{1}{a^{2}}+\frac{y}{a}\right) \\
& \times\left[\left(t+\frac{y^{2}}{2}\right) \operatorname{erfc}\left(\frac{y}{2 \sqrt{t}}\right)-\sqrt{\frac{t}{\pi}} y e^{-y^{2} / 4 t}\right] \\
& +\frac{e^{a y}}{a^{2}} \int_{0}^{t} e^{a^{2} s} \operatorname{erfc}\left(\frac{y}{2 \sqrt{s}}+a \sqrt{s}\right) d s, \\
& u_{4}(y, t)=\sqrt{\frac{t}{\pi}} \frac{y}{3}\left(y^{2}+4 t\right) e^{-y^{2} / 4 t} \\
& -y^{2}\left(\frac{y^{2}}{6}+t\right) \operatorname{erfc}\left(\frac{y}{2 \sqrt{t}}\right) \text {. }
\end{aligned}
$$

Here, $a=1 / \eta$ is a constant and $H(t-1)$ is the unit step function defined as

$$
H(t-a)= \begin{cases}0 & \text { for } 0 \leq t<a \\ 1 & \text { for } t \geq a\end{cases}
$$

It is important to note that (7) and (8), in the absence of slip effect, reduce to those obtained by Chandran et al. [6, Equations (1) and (2)].

2.1. Plate with Constant Temperature. In order to show the effect of the ramped temperature distribution of the boundary on the flow, it is necessary to compare such a flow with the one near a plate with constant temperature. The temperature and velocity variables for the flow near a plate with constant temperature can be expressed as

$$
\begin{gathered}
\theta(y, t)=\operatorname{erfc}\left(\frac{y}{2 \sqrt{t}}\right), \\
u(y, t)=\left\{\begin{array}{cc}
\frac{1}{\operatorname{Pr}-1}\left[a \sqrt{\operatorname{Pr}} u_{5}(y, t)+u_{6}(y, t)\right. \\
-F(y, t)] & \text { for } \operatorname{Pr} \neq 1 \\
\frac{1}{2}\left[y u_{7}(y, t)-a u_{5}(y, t)\right] & \text { for } \operatorname{Pr}=1,
\end{array}\right.
\end{gathered}
$$

where

$$
\begin{gathered}
u_{5}(y, t)=\frac{2}{a}\left[\sqrt{\frac{t}{\pi}} e^{-y^{2} / 4 t}-\left(\frac{1}{a^{2}}+\frac{y}{a}\right) \operatorname{erfc}\left(\frac{y}{2 \sqrt{t}}\right)\right. \\
\left.+\frac{1}{a^{2}} e^{a y+a^{2} t} \operatorname{erfc}\left(\frac{y}{2 \sqrt{t}}+a \sqrt{t}\right)\right], \\
u_{6}(y, t)=\frac{1}{\pi} \int_{0}^{\infty}\left(\frac{t}{x}-\frac{1-e^{-x t}}{x^{2}}\right) \\
\times\left[\frac{a \sin (y \sqrt{x})}{x+a^{2}}+\frac{\sqrt{x} \cos (y \sqrt{x})}{x+a^{2}}\right] d x, \\
u_{7}(y, t)=2 \sqrt{\frac{t}{\pi}}-y \operatorname{erfc}\left(\frac{y}{2 \sqrt{t}}\right) .
\end{gathered}
$$

The corresponding Nusselt number and skin friction which are, respectively, the measures of the rate of heat transfer and shear stress at the plate can be determined by considering (7) into

$$
\begin{gathered}
\mathrm{Nu}=\mathrm{Nu}(t)=-\left.\frac{\partial \theta(y, t)}{\partial y}\right|_{y=0} ; \quad t>0, \\
\mathrm{Nu}(\text { ramped })=2 \sqrt{\frac{\operatorname{Pr}}{\pi}}[\sqrt{t}-\sqrt{t-1} H(t-1)], \\
\mathrm{Nu} \text { (constant) }=\sqrt{\frac{\operatorname{Pr}}{\pi t}}
\end{gathered}
$$

and (8) into

$$
\begin{aligned}
& \tau=\tau(t)=\left.\frac{\partial u(y, t)}{\partial y}\right|_{y=0} ; \quad t>0, \\
& \tau(\text { ramped })=\frac{1}{\operatorname{Pr}-1} \\
& \times\left[I_{1}(t)-I_{2}(t)+\sqrt{\operatorname{Pr}} I_{3}(t)\right. \\
& -\left\{I_{1}(t-1)-I_{2}(t-1)+\sqrt{\operatorname{Pr}} I_{3}(t-1)\right\} \\
& \times H(t-1)] \text {, } \\
& \tau \text { (constant })=\frac{1}{\operatorname{Pr}-1}\left[\sqrt{\operatorname{Pr}} I_{4}(t)+I_{5}(t)-I_{6}(t)\right] .
\end{aligned}
$$


Here

$$
\begin{gathered}
I_{1}(t)=\frac{a t^{2}}{2}-\left[\frac{e^{a^{2} t}}{a^{3}} \operatorname{erfc}(a \sqrt{t})-\frac{1}{a^{3}}\right. \\
-\frac{t}{a}+\frac{4 t^{3 / 2}}{3 \sqrt{\pi}}+\frac{2}{a^{2}} \sqrt{\left.\frac{t}{\pi}\right],} \\
I_{3}(t)=\frac{1}{3} \sqrt{\frac{\operatorname{Pr}}{\pi} t^{3 / 2},} \frac{1}{a^{3}}\left[e^{a^{2} t} \operatorname{erfc}(a \sqrt{t})+2 a \sqrt{\frac{t}{\pi}}-a^{2} t-1\right], \\
I_{4}(t)=e^{a^{2} t} \operatorname{erfc}(a \sqrt{t})-1, \\
I_{5}(t)=t-\left[\frac{1}{a^{2}}-\frac{2}{a} \sqrt{\frac{t}{\pi}}-\frac{e^{a^{2} t}}{a^{2}} \operatorname{erfc}(a \sqrt{t})\right], \\
I_{6}(t)=-2 \sqrt{\frac{\operatorname{Pr} t}{\pi}} .
\end{gathered}
$$

\section{Results and Discussion}

The problem of heat transfer to unsteady flow of a viscous incompressible fluid with ramped wall temperature and slip condition at the wall is addressed in this study. Numerical calculations have been carried out for the dimensionless temperature $\theta$, velocity $u$, skin friction $\tau$, and Nusselt number $\mathrm{Nu}$ for the case of ramped and constant temperature of the plate. The effects of pertinent parameters such as slip parameter $\eta$, Prandtl number Pr, and time $t$ on ramped and constant profiles of temperature $\theta$, velocity $u$, skin friction $\tau$, and Nusselt number $\mathrm{Nu}$ are shown graphically. Figure 1 depicts that the velocity in case of ramped temperature plate decreases with increase of slip parameter for $y<1$ and increases for $y>1$. However, in Figure 2 velocity in case of constant temperature plate is always increasing due to increase in the values of $\eta$. In order to examine the effect of ramped temperature against constant temperature on the fluid velocity, we have plotted Figures 3 and 4 . We observe from Figure 3 that velocity in the case of ramped temperature is always less than that of velocity in case of constant temperature. This behaviour is in agreement with the graphical results from [6, 7]. Furthermore, Figures 3 and 4 also elaborate the influence of Prandtl number $\mathrm{Pr}$ and time $t$ on fluid velocity in case of ramped temperature and constant temperature. It is observed that velocity is a decreasing function of time. However, it is further noticed that velocity near the plate is greater and is continuously decreasing with increasing distance from the plate and finally approaches to zero for large values of $y$. It is also observed that the velocity of the fluid is greater for air $(\operatorname{Pr}=0.71)$ than that of water $(\mathrm{Pr}=7.0)$. Figure 5 is prepared for the constant velocity profile for different values of time $t$ when $\eta=0.02$ and $\operatorname{Pr}=0.71$. This figure clearly shows that when time is zero, the velocity satisfies initial condition given in (6).

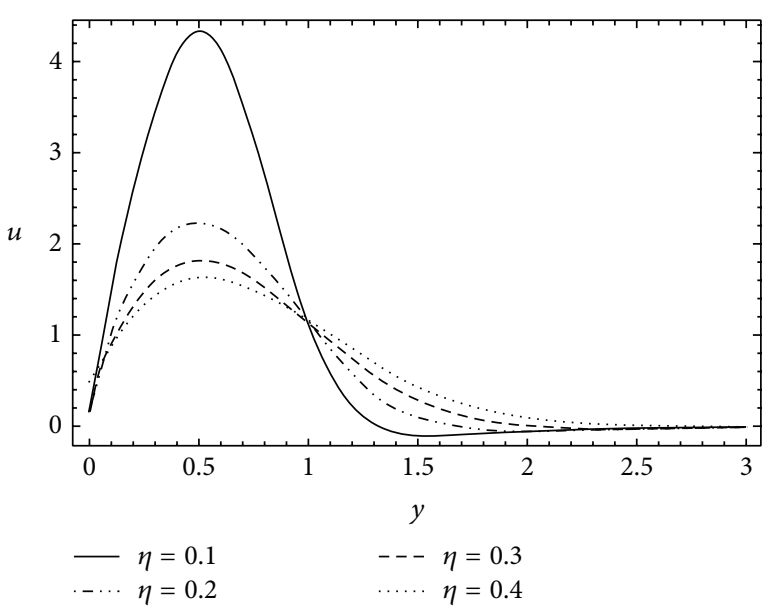

FIGURE 1: Velocity profiles for different values of $\eta$ corresponding to ramped temperature of the plate with $t=0.9(\mathrm{Pr}=0.71)$.

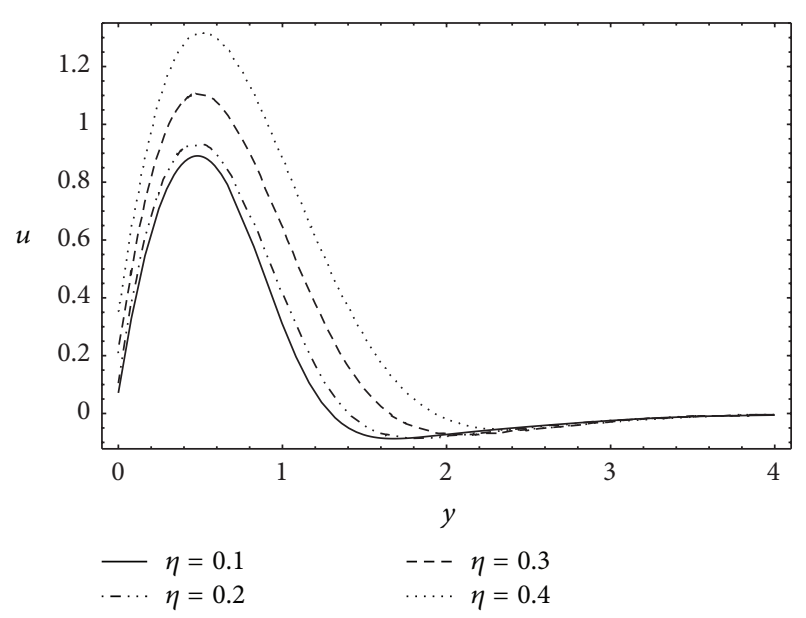

FIGURE 2: Velocity profiles for different values of $\eta$ corresponding to stepped temperature of the plate with $t=0.9(\operatorname{Pr}=0.71)$.

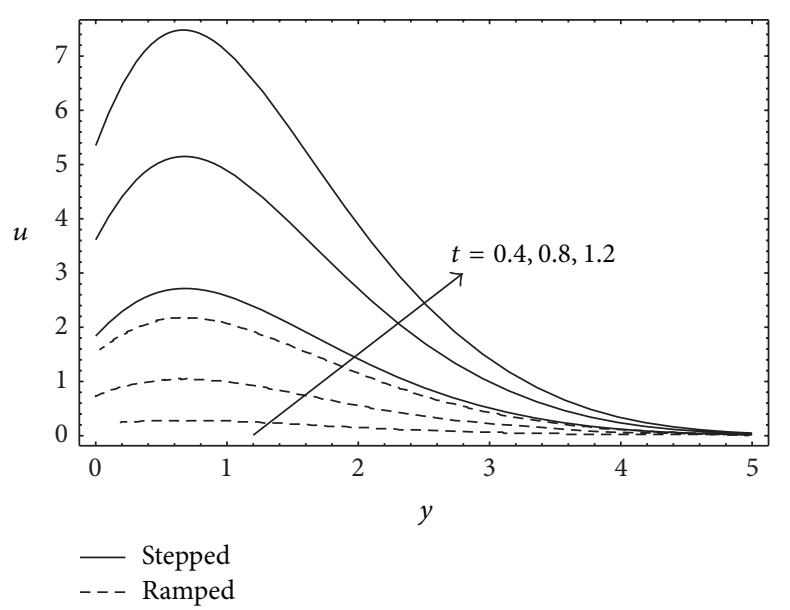

FIGURE 3: Velocity profiles for different values of $t$ with $\eta=0.8$ and $\operatorname{Pr}=0.71$. 


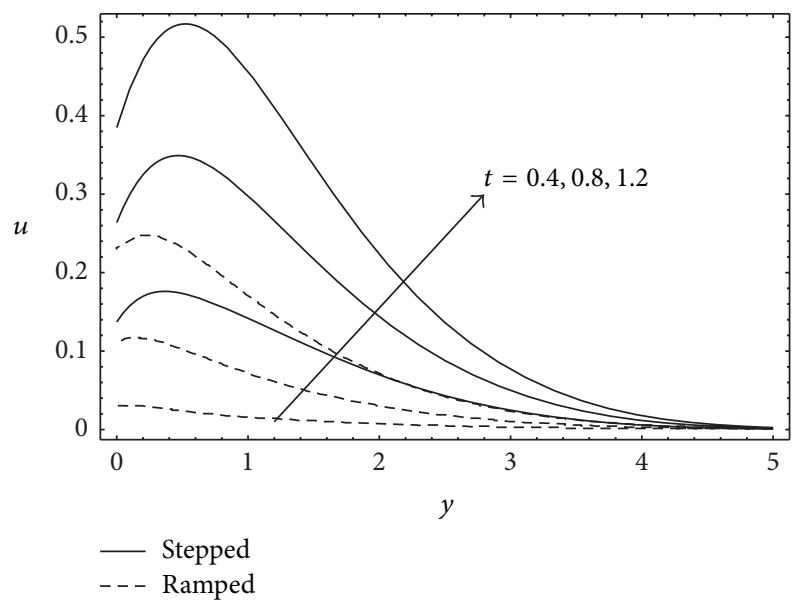

Figure 4: Velocity profiles for different values of $t$ with $\eta=0.8$ and $\operatorname{Pr}=7.0$.

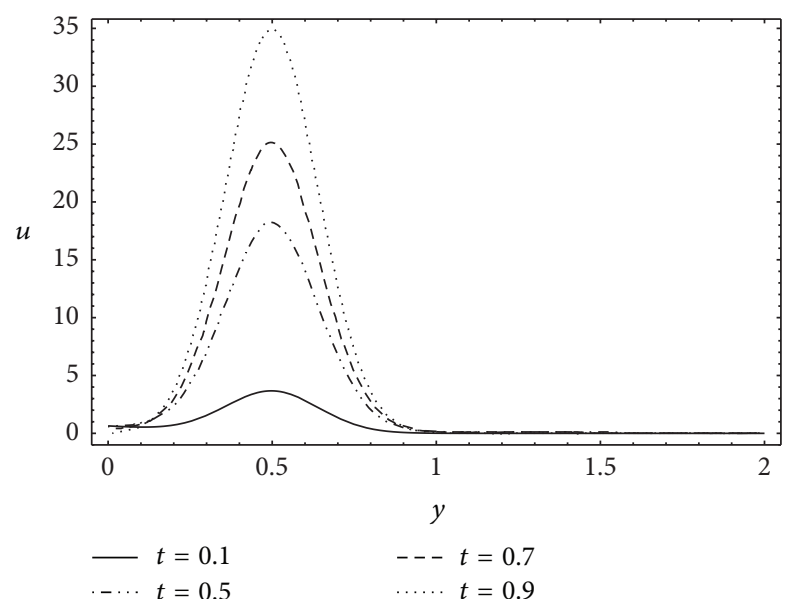

Figure 5: Velocity profiles for different values of $t$.

Figures 6 and 7 illustrate the variations of temperature profiles for different values of $\operatorname{Pr}$ and $t$. Two different values of $\operatorname{Pr}$ such as $\operatorname{Pr}=0.71$ and 7.0 are chosen. It is depicted from Figure 6 that temperature profiles decrease with increasing values of $\mathrm{Pr}$, both in the case of ramped and constant temperature at the plate. It is observed that the thermal boundary layer thickness is maximum near the plate and decreases with increasing distance from the leading edge and finally approaches zero. Furthermore, ramped temperature profiles are found smaller than constant profiles of the temperature. It is observed from Figure 7 that temperature increases with an increase in time in the case of ramped as well as constant temperature at the plate. From these graphs, it is clearly seen that, for ramped wall temperature case, the temperature takes the values of time at the plate boundary whereas its values is one for all $t>1$. Hence these graphs show that temperature profiles satisfy the imposed boundary conditions in (6).

The skin friction variations along time $t$ are shown in Figures 8 and 9 for different values of slip parameter $\eta$. Figure 8 shows that skin friction is decreasing with increasing

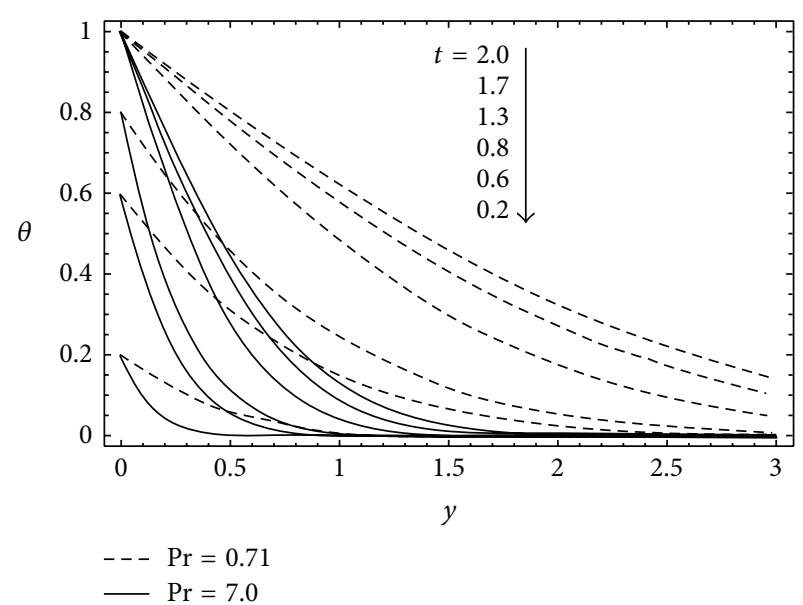

Figure 6: Temperature profiles for two different values of Pr.

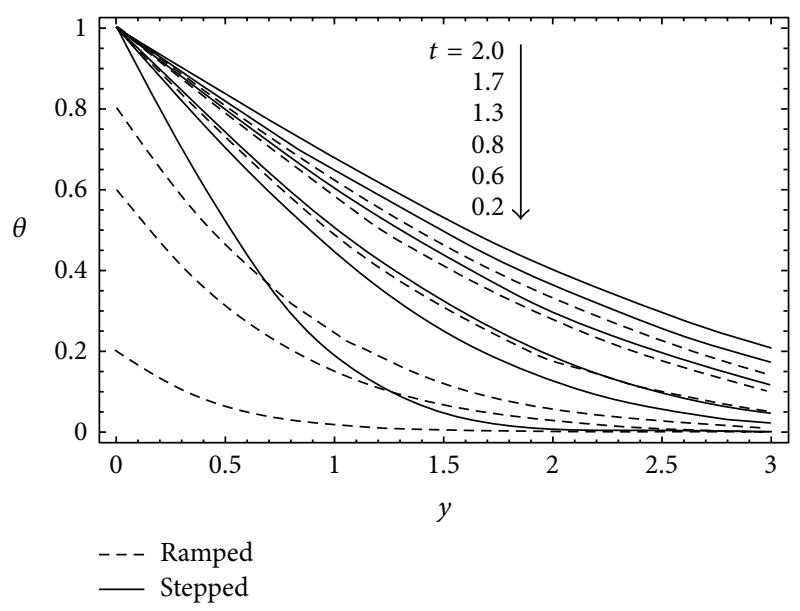

Figure 7: Temperature profiles for different values of $t$.

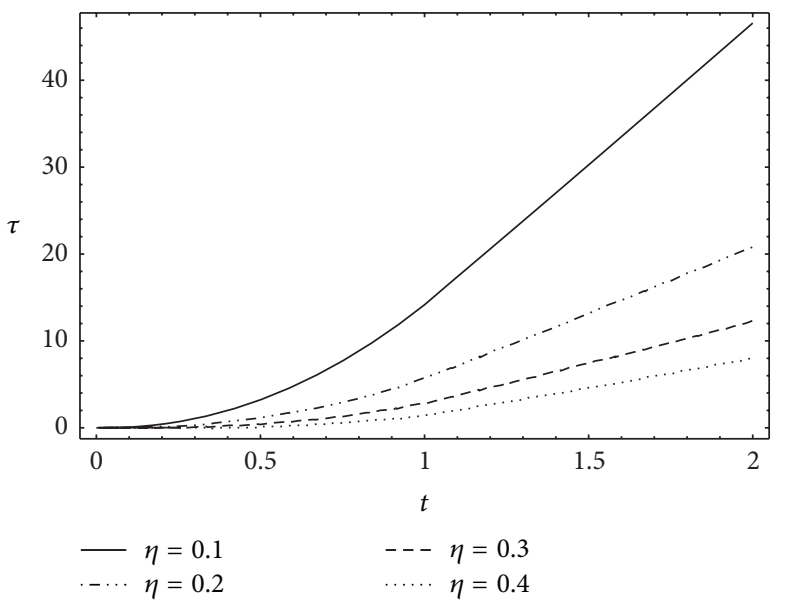

FIGURE 8: Variations of skin friction $\tau$ along $t$ for different values of $\eta$ corresponding to ramped temperature of the plate with $\operatorname{Pr}=0.71$. 


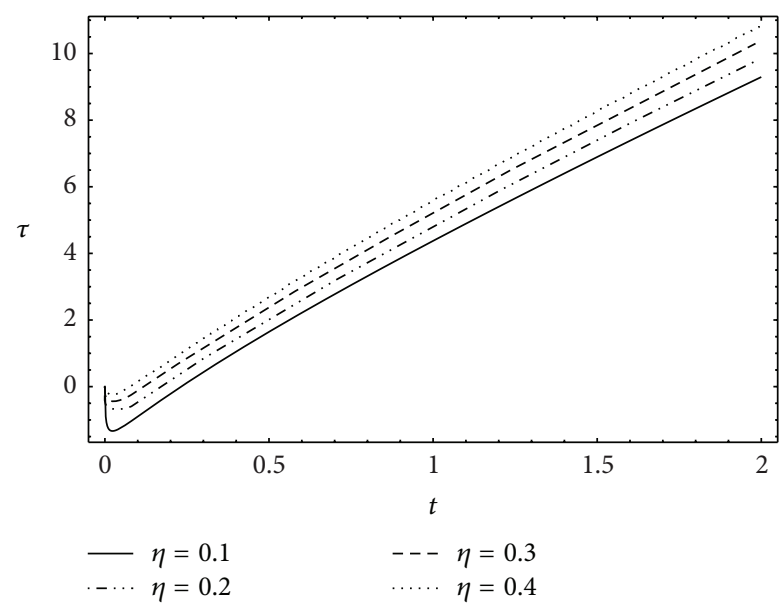

FIGURE 9: Variations of skin friction $\tau$ along $t$ for different values of $\eta$ corresponding to stepped temperature of the plate with $\operatorname{Pr}=0.71$.

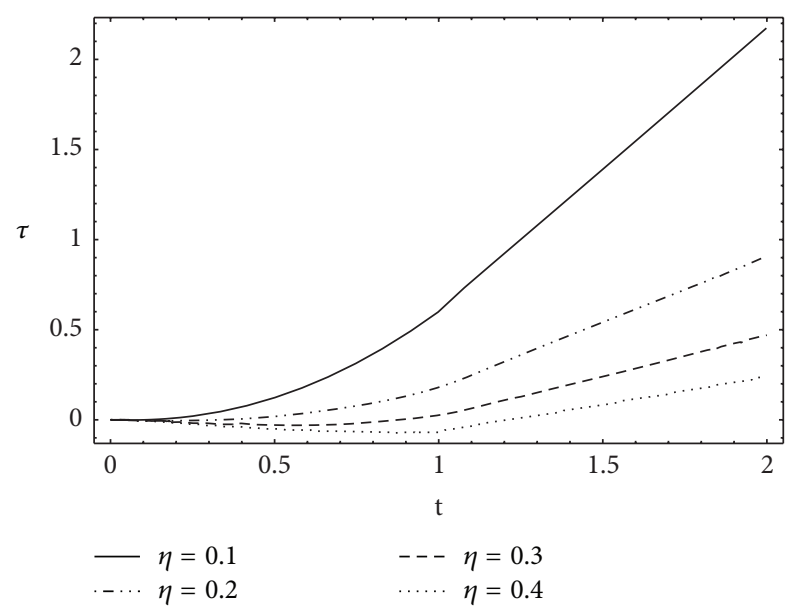

FigURE 10: Variations of skin friction $\tau$ along $t$ for different values of $\eta$ corresponding to ramped temperature of the plate with $\operatorname{Pr}=7.0$.

values of $\eta$ in case of ramped temperature of the plate, while, in Figure 9, we observe that skin friction is increasing due to increase in $\eta$ in case of constant temperature of the plate. From the comparison of Figures 8 and 10 we deduce that skin friction decreases due to the increase in Prandtl number Pr in case of ramped temperature of the plate. Again from the comparison of Figures 9 and 11, we conclude that skin friction is decreasing due to the increasing values of Prandtl number Pr in case of constant temperature of the plate. The variation of Nusselt number is shown in Figures 12 and 13 for different values of Pr. Figures 12 and 13 depict that Nusselt number increases with increasing Pr, both in case of ramped and constant temperatures of the plate. It is further noted that Nusselt number for water $(\operatorname{Pr}=7.0)$ is greater than electrolytic solution ( $\mathrm{Pr}=1.0)$, air $(\operatorname{Pr}=0.71)$, and mercury $(\operatorname{Pr}=0.015)$. Physically, it is justified due to the fact that large values of Prandtl are responsible to decrease the thermal conductivity and therefore heat diffuses more slowly from the plate than for smaller values of Pr. Hence the rate of heat

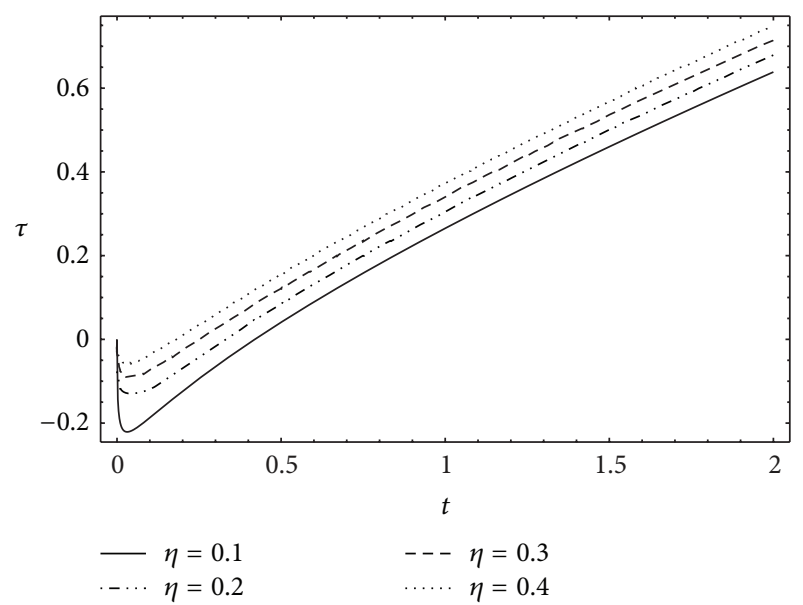

FIGURE 11: Variations of skin friction $\tau$ along $t$ for different values of $\eta$ corresponding to stepped temperature of the plate with $\operatorname{Pr}=7.0$.

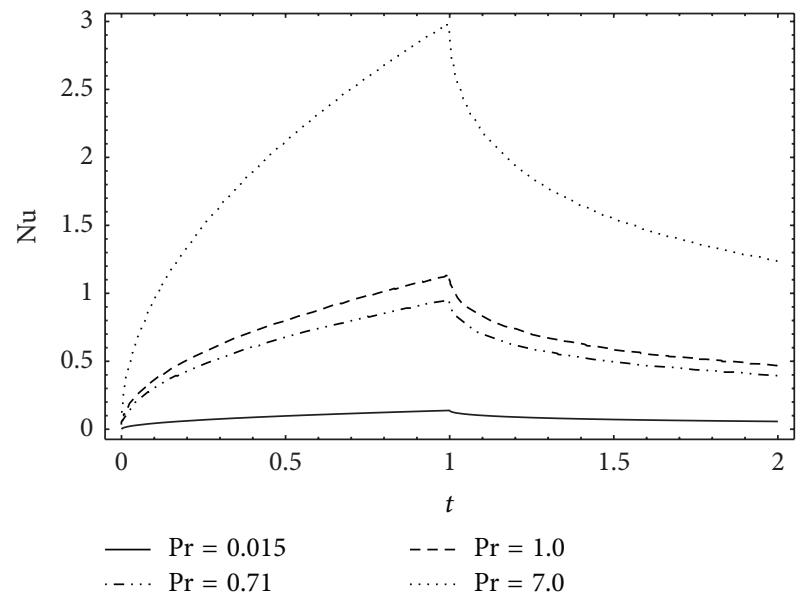

FIGURE 12: Variations of Nusselt number Nu along $t$ for different values of Pr corresponding to ramped temperature of the plate.

transfer is increased. This behavior is in a proper agreement with the observations made in [6, Figure 6].

\section{Conclusions}

The influence of slip condition on free convection flow of an incompressible viscous fluid past a vertical plate with ramped wall temperature is investigated. Laplace transform procedure is used for finding the exact solutions of the problem. The expressions for velocity and temperature are obtained in terms of the exponential and complementary error functions. It is found that they satisfy all the imposed initial and boundary conditions and as a special case can be reduced to the similar solution existing in the literature. The effects of different physical parameters such as slip parameter $\eta$, Prandtl number Pr, and time $t$ on ramped and constant profiles of temperature, velocity, skin friction, and Nusselt number are studied graphically. It is observed that the velocity increases with increasing slip parameter $\eta$, whereas 


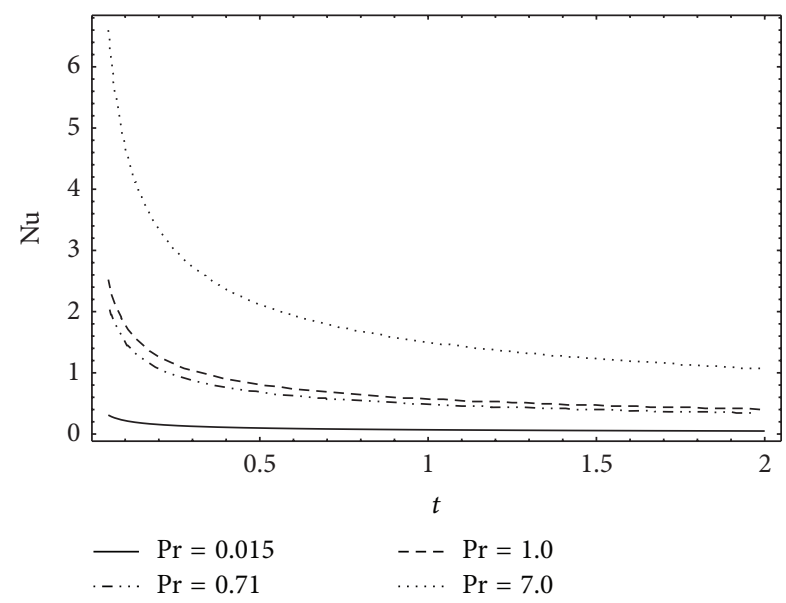

FIgURE 13: Variations of Nusselt number $\mathrm{Nu}$ along $t$ for different values of Pr corresponding to stepped temperature of the plate.

it decreases with increasing time. Temperature in case of air is greater than water and decreases with increasing Pr and $t$. Skin friction increases when the temperature is constant while it decreases for the ramped nature of temperature. Nusselt number increases in both cases of constant and ramped temperatures.

\section{Conflict of Interests}

The authors declare that there is no conflict of interests regarding the publication of this paper.

\section{References}

[1] R. Siegel, "Transient free convection from a vertical flat plate," Transactions of the American Society of Mechanical Engineers, vol. 80, pp. 347-359, 1958.

[2] J. A. Schetz, "On the approximate solution of viscous flow problems," ASME Journal of Applied Mechanics, vol. 30, pp. 263$268,1963$.

[3] A. A. Hayday, D. A. Bowlus, and R. A. McGraw, "Free convection from a vertical flat plate with step discontinuities in surface temperature," Journal of Heat Transfer, vol. 89, no. 3, pp. 244249, 1967.

[4] C. P. Malhotra, R. L. Mahajan, W. S. Sampath, K. L. Barth, and R. A. Enzenroth, "Control of temperature uniformity during the manufacture of stable thin-film photovoltaic devices," International Journal of Heat and Mass Transfer, vol. 49, no. 17-18, pp. 2840-2850, 2006.

[5] K. A. Antonopoulos and F. Democritou, "Experimental and numerical study of unsteady non-periodic wall heat transfer under step, ramp and cosine temperature perturbations," International Journal of Energy Research, vol. 18, no. 6, pp. 563-579, 1994.

[6] P. Chandran, N. C. Sacheti, and A. K. Singh, "Natural convection near a vertical plate with ramped wall temperature," Heat and Mass Transfer, vol. 41, no. 5, pp. 459-464, 2005.

[7] G. S. Seth, M. S. Ansari, and R. Nandkeolyar, "MHD natural convection flow with radiative heat transfer past an impulsively moving plate with ramped wall temperature," Heat and Mass Transfer, vol. 47, no. 5, pp. 551-561, 2011.

[8] M. Narahari, O. A. Bég, and S. K. Ghosh, "Mathematical modelling of mass transfer and free convection current effects on unsteady viscous flow with ramped wall temperature," World Journal of Mechanics, vol. 1, no. 4, pp. 176-184, 2011.

[9] D. C. Tretheway and C. D. Meinhart, "Apparent fluid slip at hydrophobic microchannel walls," Physics of Fluids, vol. 14, no. 3, pp. L9-L12, 2002.

[10] O. D. Makinde and E. Osausi, "MHD steady flow in a channel with slip at the permeable boundaries," Romanian Journal of Physics, vol. 51, pp. 319-328, 2006.

[11] O. D. Makinde and P. Y. Mhone, "Heat transfer to MHD oscillatory flow in a channel Ölled with porous medium," Romanian Journal of Physics, vol. 50, pp. 931-938, 2005.

[12] A. Mehmood and A. Ali, "The effect of slip condition on unsteady MHD oscillatory flow of a viscous fluid in a planer channel," Romanian Journal of Physics, vol. 52, pp. 85-92, 2007.

[13] A. M. Rohni, S. Ahmad, I. Pop, and J. H. Merkin, "Unsteady mixed convection boundary-layer flow with suction and temperature slip effects near the stagnation point on a vertical permeable surface embedded in a porous medium," Transport in Porous Media, vol. 92, no. 1, pp. 1-14, 2012.

[14] T. Hayat, S. A. Shehzad, and M. Qasim, "Slip effects on the unsteady stagnation point flow with variable free stream," Walailak Journal of Science and Technology, vol. 10, no. 4, pp. 385-394, 2013.

[15] M. Qasim, Z. H. Khan, W. A. Khan, and I. Ali Shah, "MHD boundary layer slip flow and heat transfer of ferrofluid along a stretching cylinder with prescribed heat flux," PLOS ONE, vol. 9, no. 1, Article ID e83930, 2014.

[16] E. A. Ashmawy, "Fully developed natural convective micropolar fluid flow in a vertical channel with slip," Journal of the Egyptian Mathematical Society, 2014. 


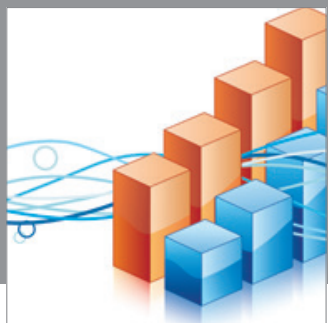

Advances in

Operations Research

mansans

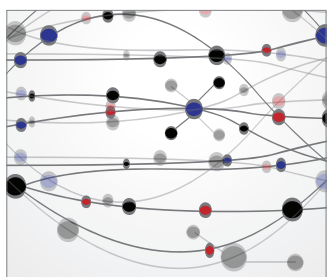

The Scientific World Journal
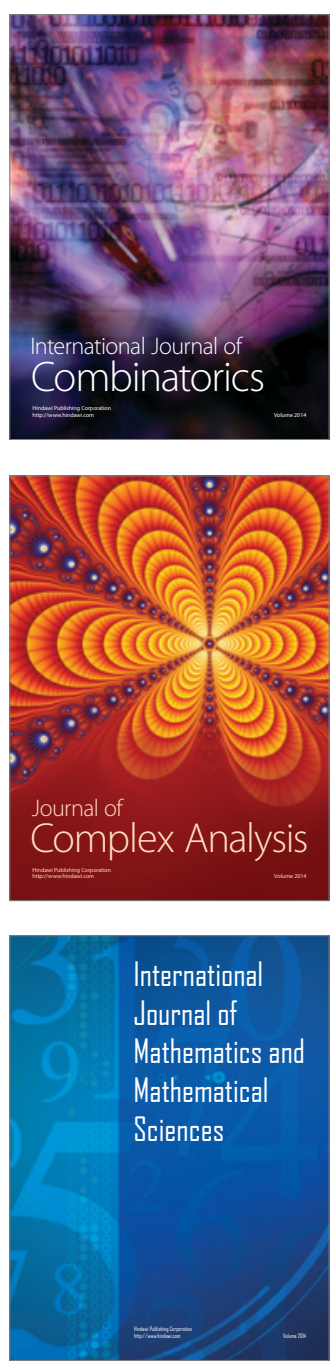
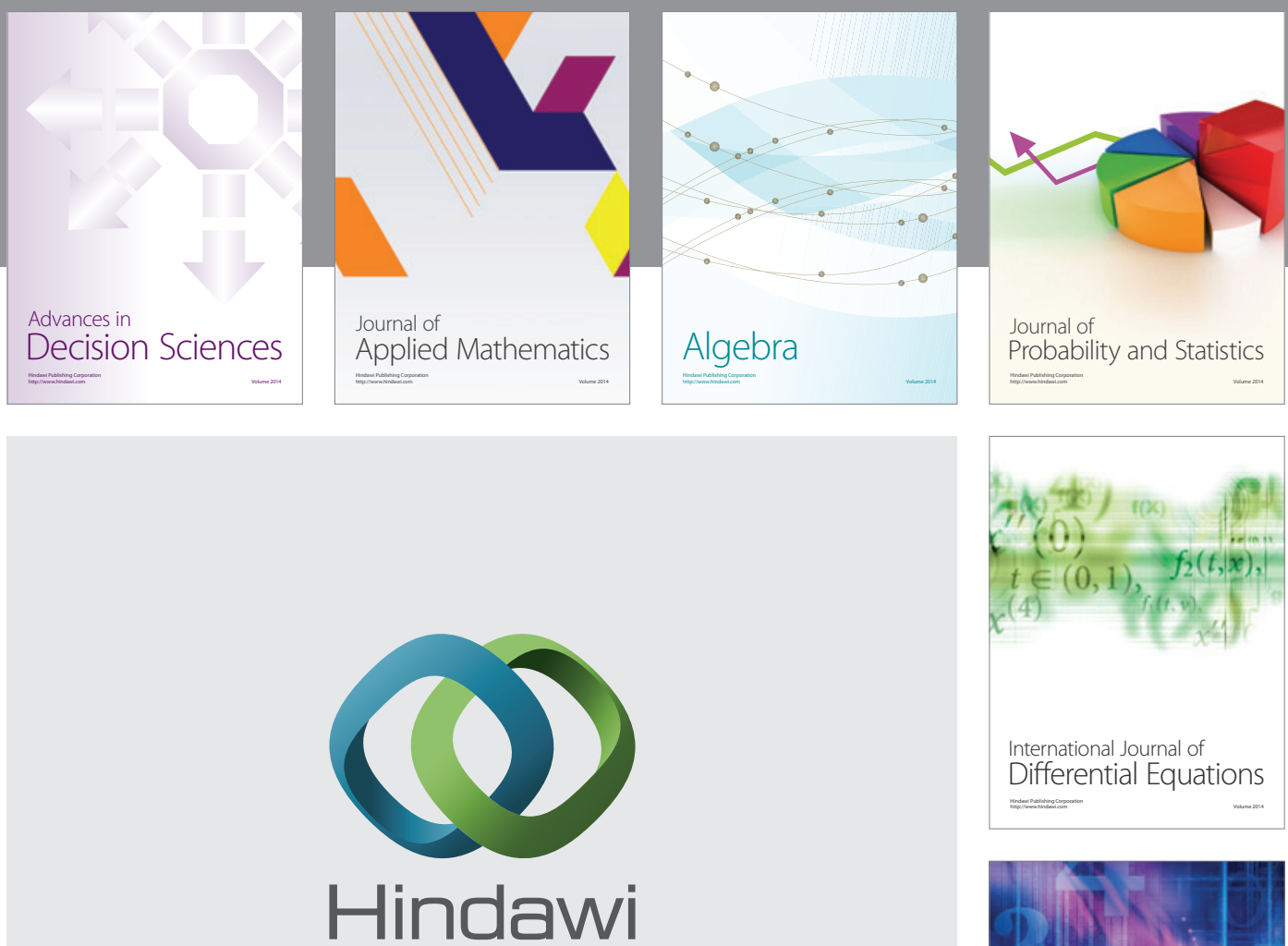

Submit your manuscripts at http://www.hindawi.com
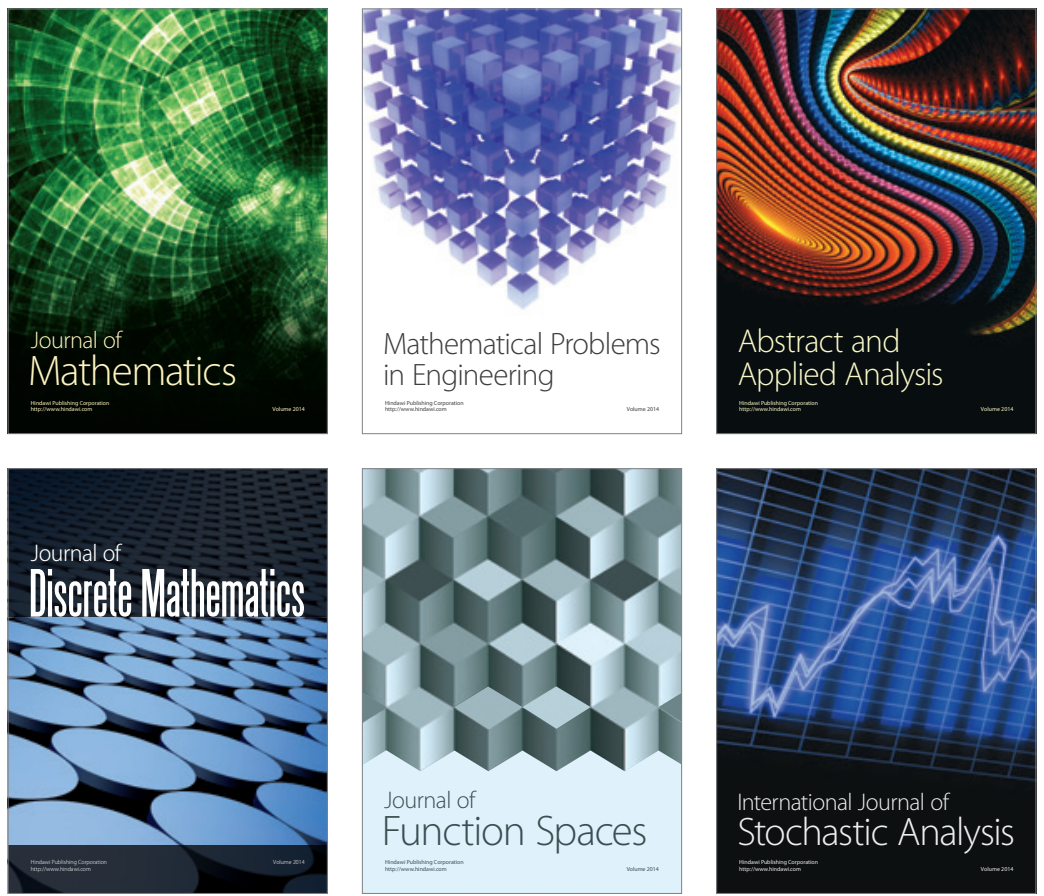

Journal of

Function Spaces

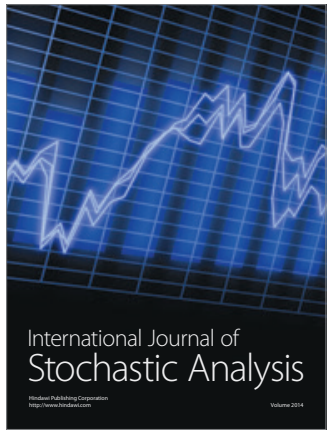

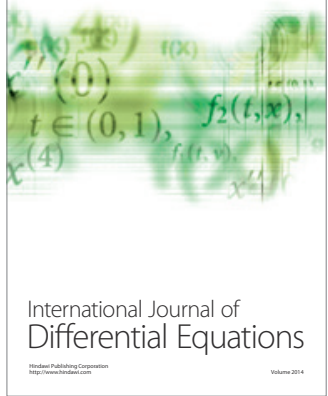
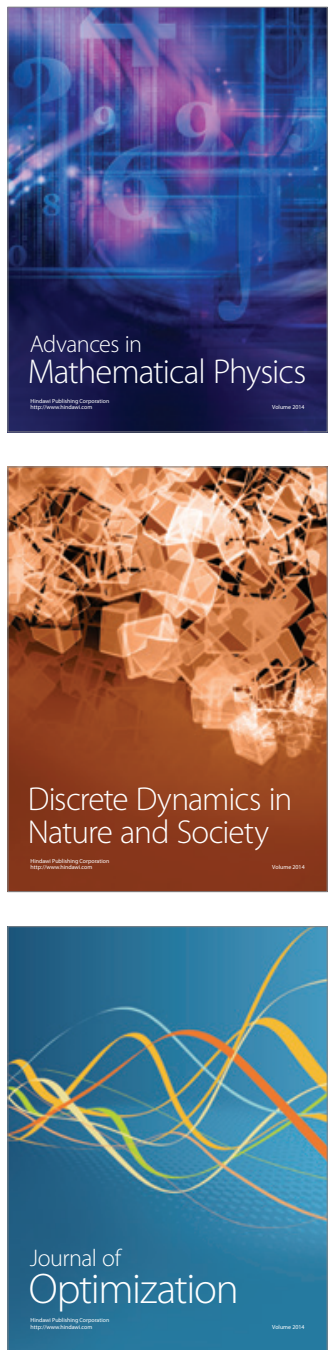